\title{
Local min.-energy, F E based formulation for well- path design
}

\author{
Kailash Jha \\ IIS, Scientific Computing Ltd. \\ New Delhi, India
}

Abstract: A new algorithm has been developed in this work to generate an optimal well path for drilling applications. Minimum energy based finite element technique has been used for interpolating well path. The straight portion of the well path at beginning and end of segment has been maintained. The dog-length severity has been maintained in present interpolation technique.

Present algorithm will give total length of the well as well as maximum value of the doglength severity. These two parameters are useful to estimate the cost of drilling.

Key words: B-splines, interpolation. minimal energy, chord length parameterization, dog-length severity, well planning, horizontal well, directional drilling.

\section{INTRODUCTION}

In present work minimum energy based curve fitting has been done for horizontal well path design (see Joshi [1]). A brief overview of horizontal well design is given in reference [1]. In early days of oil drilling, only vertical wells were drilled. These types of wells can not be optimal wells because of poor flowability and their simple geometry. Poor flowability results in low productivity and simple geometry restricts the approach to complicated reservoir. If there is any obstacle in direction of drilling then drilling process has to be restarted. This leads to a huge wastage of money 
and time. These difficulties have been overcome in directional drilling. Directional drilling may be of horizontal shape. In general directional drilling can take any shape. Obstacles in the direction of drilling may be due to presence of salts or faults. If there is salt or fault in direction of drilling it is very hard for drill bit to cross it. This is true for any types of drilling. Another importance of directional drilling is to enhance the reservoir contacts and better productivity. A long path in direction of reservoir gives a larger contact area and hence enhances the injectivity. There are basically two types of non-vertical wells

(1) Deviated and (2) Horizontal

Deviated drilling applies to a well, which is designed to reach a particular point in a reservoir, which is substantially different from the surface location

(2) Horizontal drilling applies to a well, which is designed to enter a reservoir roughly parallel to formation boundaries and remain there for some distance.

A non-linear optimization for well design has been discussed in reference [2]. In this work, optimal parameters have been calculated and compared with actual trajectory of the well.

In the present work an attempt is made to get the optimal well path by iterative design. An optimal bore well path has minimal length for pre-specified minimum radius of curvature as well as minimum total energy. Total length of the well for a given minimum radius of curvature has been calculated in this work. Total length of well includes the straight portions and the curved portions of the well. In any segment of well path, if the minimum value of radius of curvature is less than given minimum value, the energy parameters are rectified to get the required condition. Doglength severity is measurement of magnitude of radius of curvature.

Drilling parameters can be changed to get the required minimum radius of curvature. By iterative technique optimal drilling parameters can be obtained. Total length and minimum radius of curvature are very important to calculate total cost of drilling. There are four coefficients, which have been used to control smoothness of the well path. For each segment of the well bore, pre and post hold lengths are specified. In present energy based technique an attempt is made to achieve the straight-line requirement of the borehole at starting and end of the segment by current interpolation technique.

In the proposed research work, points on well path, pre-hold and posthold lengths are the prime inputs. Tangents are optional. If tangents are not given, 3-point method [3] is used to get tangent values. Points and tangents give geometry and direction of well path for a segment. Pre-hold and posthold lengths give the straight portion of the well path. Cubic B-Spline curve 
fitting based on local minimal energy has been developed to get required well path. This technique assures matching of input parameters. For each segment of the well path, three elements have been considered to get finite element based minimum energy formulation. First and third elements of a segment are straight lines where as middle portion (second element of the segment) has curved shape. For each element different coefficients can be considered according to the requirements. In straight portion, energy coefficients are put to zero. In curved portion of the well suitable values of coefficients have been used. Effect of these coefficients is studied in this work to get an optimal well.

While designing a well path, it is required that the well profile should be smoother, radius of curvature should be more than given value and total length should be as minimum as possible. Higher order derivatives also can be considered in constrained equation.

This paper has been organisation as follows:

Section-2 contains a brief literature survey of energy based curve fitting as well as well path design. Current work has been explained in section-3. In section-4, results of implementations have been discussed along with comparison with previous works. Conclusions have been made in section-5.

\section{RELATED WORK}

There are number of researchers who worked on the principle of minimum energy to fit a curve through set of points [4-12]. Early researchers have tried to approximate total energy of curve/surface by equation- 1

$$
\int_{t} K^{2}(t) d t
$$

Where $\mathrm{K}(\mathrm{t})$ is curvature function and $\mathrm{t}$ is parametric value.

Kjellander[4] and Lee[6] used approximation of eqation-1 for splines. Kallay [5] used discrete approximation to solve equation-1 for given length. Cheng and Barsky[7] used energy-based spline fit to pass through specific regions. This is useful when some of fitting points are uncertain. Vassilev[9] used energy technique for fairing curve and surfaces. Result obtained by approximating curvature function $(\mathrm{K}(\mathrm{t}))$ does not approximate the curve correctly (see Wang et al [8]). It may be suitable for certain specific requirements. Wang et al [8] has given different approximations and compared the results. They have used different parameterisation techniques like (a) uniform, (b) chord length and (c) centripetal to get different results. 
They found that energy has a bigger impact in generation of curves and surfaces than parametric technique in curve or surface interpolation. They also found that approximation of energy would not approximate the curve/surface properly. A brief description of energy based formulation is given in Park et al. [10]. Well design concept has been explained in Joshi [1]. In this reference a brief description of horizontal well technology has been done. Comparison with vertical well has been performed effectively in this work. Concept of optimisation is explained in Helmy [2]. In this work nonlinear optimisation has been done to get optimal drilling parameters.

I adopted similar terms as used in references [10-12] in my energy function. In present work, one more energy term has been used than reference [10], which is responsible for smoother curvature (see equation-2). This term

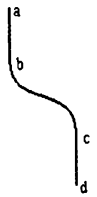

Fig. 1(conventional S-type well)

involves the third derivatives. In present approach local minimization technique has been adopted to ensure the present requirement of fix pre and post hold lengths. Pre and post hold lengths are the straight portions of well segment which are shown in the Figure-1. ab and cd are the straight portions of the well segment shown in Figure-1 which are called pre and post hold lengths. Curved portion of well segment is bc. Points and tangents are defined at segment points a and $d$ respectively. Tangent directions for this segment are $a b$ and $c d$. Proposed work solves quadratic functional minimization where a resulting curve can be obtained by solving a linear set of equations see [10].

\section{CURRENT WORK}

In this paper an energy based interpolation technique has been developed to get a smooth well path in such a way that it has minimum energy.

Total energy considered in this formulation is given by equaition-2, which is given below: 


$$
\begin{aligned}
E=\int_{t} \alpha\left(C_{t}(t) \cdot C_{t}(t)\right) & +\int_{t} \beta\left(C_{t t}(t) \cdot C_{t t}(t)\right)+\int_{t} \gamma\left(C_{t t t}(t) \cdot C_{t t t}(t)\right) \\
& +\gamma 1 \sum_{i=0}\left\|P_{i}-C\left(t_{i}\right)\right\|^{2}
\end{aligned}
$$

Where $\alpha, \beta$ and $\gamma 1$ are non-negative value called stretching, bending and fitting coefficient respectively.

$\gamma$ is a new non-negative coefficient of energy responsible for having smooth curvature.

$\mathrm{C}(\mathrm{t})$ is required cubic B-Spline curve.

$\mathrm{P}_{\mathrm{i}}$ denotes given points.

$C_{t}, C_{t t}$ and $C_{t t t}$ are first, second and third derivations of curve $C(t)$.

$t$ is parametric value. These energy terms have been explained in [10-12]. Effect of these coefficient have been studied in present work. I adopted a quadratic functional minimization technique, which results in a curve by solving linear set of equations see Park et al [10]. Assuming equation-3 given below to be solution of equation-2.

$\mathrm{C}(\mathrm{t})=\sum \mathrm{N} \mathrm{X}$

Where $\mathrm{N}$ represents basis functions and $\mathrm{X}$ represents Control points (see Piegl and Tiller [3]) in equation-3. Equation -2 can be rewritten as given below:

$$
\begin{aligned}
& X^{T}\left(\alpha N_{t} N_{t}^{T}+\beta N_{t t} N_{t t}{ }^{T}+\gamma N_{t t t} N_{t t t}{ }^{T}\right)+\gamma 1(A X-P)^{T}(A X-P)- \\
& \Rightarrow X^{T}(\alpha K 1+\beta K 2+\gamma K 3) X+\gamma 1(A X-P)^{T}(A X-P)
\end{aligned}
$$

Where $\mathrm{N}_{\mathrm{t}}, \mathrm{N}_{\mathrm{tt}}, \mathrm{N}_{\mathrm{ttt}}$ represents first, second and third derivatives of Basis Function see Piegl and Tiller [3]. K1, K2 and K3 are $4 \times 4$ matrices see $[8,12$ ]. $\mathrm{A}$ and $\mathrm{P}$ are representing coefficient matrix and input points respectively. Constraints are given by equation -5 below:

$$
\mathrm{C}_{\mathrm{k}} \mathrm{X}=\mathrm{D}_{\mathrm{k}}
$$

Where $\mathrm{k}$ varies from 0 to one less than number of constraints The final matrix equations can be written as given below (see[10]):

$$
\left(\begin{array}{cc}
\mathrm{K}+\gamma 1 \mathrm{~A}^{\mathrm{T}} \mathrm{A} & \mathrm{C}^{\mathrm{T}} \\
\mathrm{C} & \phi
\end{array}\right)\left({ }_{\text {here } \mathrm{V} \text { is a vector storing Langrange Multiplier. }}=\left(\begin{array}{c}
\gamma 1 \mathrm{~A}^{\mathrm{T} P} \\
\mathrm{D}
\end{array}\right)\right.
$$


$\mathrm{K}$ is a segment stiffness matrix with size $(\mathrm{nC} \mathrm{x} \mathrm{nC}) . \mathrm{nC}$ indicates number of control points.

$\mathrm{C}$ is (nconstrats $\mathrm{x} \mathrm{nC}$ ) matrix.

nconstrats indicates number of constraints given.

Above matrix equations can be solved by Gauss-elimination to get control points.

The interpolation technique is applied locally to maintain linearity requirement at the start and end of the segments and uses the contribution of energy from the third derivative (see Fang and Gossard [12]) which is given in equation-2. In current work linearity requirement for segment is overcome by putting the energy coefficients ( $\alpha, \beta$ and $\gamma$ ) for straight element as zero. The term local interpolation indicates that all points for a segment are derived from input parameters (points, tangents, pre and post hold lengths) for a segment.

In the present implementation, for each segment, eight points have been considered along with four tangents at start and end of the straight portions of each segment. Three elements have been considered for each segment. First and last elements are straight lines. Energy coefficients are kept zero to maintain linearity for straight element of the segment. Points are exactly interpolated in this work and minimum value of the radius of curvature is maintained in each segment as well as for entire well path. If any segment violates the minimum radius of curvature it will be indicated by present formulation. Once this mismatch occurs, the energy coefficients for curved portion are modified. $\gamma 1$ value reversibly affects the smoothness of the curve. Magnitude of the tangents is assumed as total arc length for the segment, which may effect the nature of curve. Parameterization is also effecting the nature of curve see[8,9]. This formulation is useful in drilling applications where the well path is highly directional. There may be situations when a drill bit will not move forward due to presence of faults or salts. In such situations, diverting the well path is required in real time while still meeting some overall requirements. The simple output of the program is shown from Figure-2 to Figure-9. Final output of the program will give a well survey points. Real wells also have been tested in this work.

\section{RESULTS AND DISCUSSION}

Results for different wells have been shown from Figure-2 to Figure-9 using OpenGL on a PC using Windows operating system. Figure-2 to Figure-6 shows results for test example-1. Figures 7 and 8 show results for test example-2 and Figure-9 shows result for test example-3. 
Boreholes are approximated as small solid cylinders. Well paths are shown as lines. Curvature is plotted against the parametric value. $\mathrm{Y}$-axis shows magnitude of curvatures magnified by 2000 and $\mathrm{X}$-axis shows parametric values with magnifying factor 1000 . Current observation shows that the affect of third part of energy term is also important along with other terms in energy based curve fitting. Fang and Gossard [12] have expressed its importance. It has been found in current work that as the value of the $\gamma$ increases the maximum fitting error and smoothness increase and total length of the well path decreases (see Table-1 and first two rows of Table-2). Table1 shows twenty sets of coefficients $(\alpha, \beta, \gamma 1$ and $\gamma)$ in favor of above statement about $\gamma$ for a segment shown in Figure-7. In well industry, it is

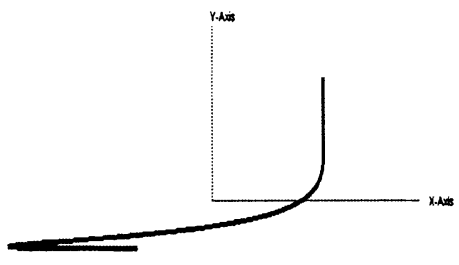

Fig. $2\left(\right.$ well $\left(\alpha=\beta=\gamma=0.0\right.$ and $\left.\left.\gamma 1=1.0 \mathrm{e}^{6}\right)\right)$

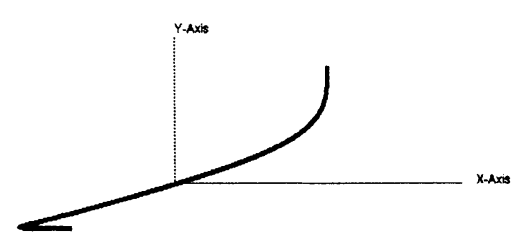

Fig 3(same well as Fig-2, except $\gamma=$.0455)

important that a well should be of minimal length as well as maximum possible radius of curvature. Figure- 2 and Figure- 3 show the borehole for a segment, for two different sets of coefficients. Figure- 4 shows the curvature plots for these bore holes. In Figure-2, values of coefficients $(\alpha, \beta, \gamma 1$ and $\gamma)$ are $0.0 .0 .0,1.0 \mathrm{e}^{6}$ and 0.0 respectively. In Figure-3, these
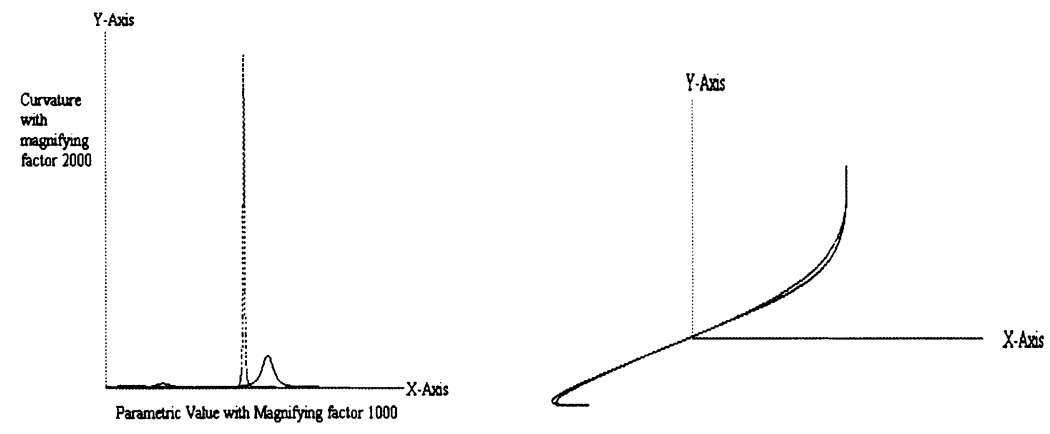

Fig. 4 (Curvature plots for figures 1,2) Fig. 5 (well paths to show the effect of $\alpha / \beta$ )

coefficients are $0.0,0.0,1.0 \mathrm{e}^{6}$ and .0455 respectively. Black solid line in Figure-4 shows curvature plot for the borehole shown in Figure-2. The blue 
dashed line in Figure- 4 shows the curvature plot of the well bore shown in Figure-3. If ratio of $\alpha$ to $\beta$ is greater than one then stretch on the well path will be effective otherwise bending will be effective (see Figure-5). If ratio of $\alpha$ to $\beta$ is more than one it increases fitting error as well as smoothness of the curve and decreases total length of the well path. If it is less than one, it decreases the fitting error and smoothness of the curve as well as increases total length. Figure-5 shows two well paths for different set of coefficients for the segment shown in Figures 1 and 2. Third and fourth rows of Tables-2 show the effect of $\alpha$ and $\beta$. Effect is measured in terms of total length, maximum curvature and maximum fitting error. The dotted blue line shows the stretch effect. In this case, $\alpha, \beta . \gamma 1$ and $\gamma$ have value $1.0, .2,1.0 \mathrm{e}^{6}$ and 0.0 respectively. In the same figure with black line shows the effect of dominance of $\beta$ value, which results more bending. Figure- 6 shows the curvature plots of the well paths shown in Figure-5.

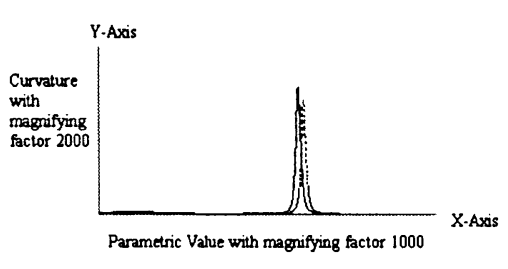

Fig. 6 (curvature plots for different $\alpha / \beta$ )

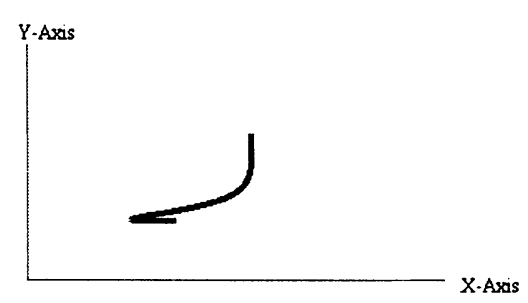

Fig. 7(bore well for test example-2)

Smooth behavior of dotted blue line shows the effect of dominance of $\alpha$ on $\beta$. In this case curvature plot are smoother. In the same figure black line shows that, higher beta dominance results in comparatively non-smoother path. It has been found in present observation that there should be a maximum limit for $\alpha$ to $\beta$ ratio for a smooth interpolation. I have achieved a good result up to a maximal value 5 .

Figure-7 shows another well segment whose curvature is plotted in Figure- 8 to show the effect of $\gamma 1$ (fitting coefficient). Blue dotted line of Figure- 8 shows the curvature plot for a segment having $\alpha, \beta, \gamma 1$ and $\gamma$ values 1.0, .2, $1.0 \mathrm{e}^{4}$ and .0455 respectively. The black solid line shown in the same figure, shows the curvature plot for the same segment having $\alpha, \beta, \gamma 1$ and $\gamma$ values $1.0, .2,1.0 \mathrm{e}^{6}$ and .0455 respectively. Curvature plots show that as the $\gamma 1$ value increases, fairness of the well path decreases. As the $\gamma 1$ value increases 
maximum fitting error decreases and total length increases (see $5^{\text {th }}$ and $6^{\text {th }}$ rows of Table-2). Figure-9 shows a real well with coefficients 0.0 ,

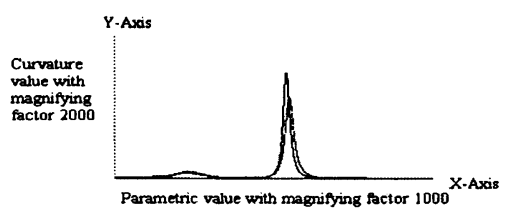

Fig. 8(curvature plots for example-2)

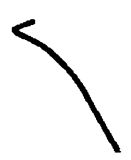

Fig. 9(real well bore example-3)

$0.02,1.0 \mathrm{e}^{6}$ and 0.0 respectively. Table $-2\left(7^{\text {th }}\right.$ and $8^{\text {th }}$ rows $)$ shows comparison of this well with well obtained by least square fit. Real effect of energy terms can be realized well in isolation of the particular coefficient. As the value of beta increases total length as well as fitting error increases and the smoothness will be enhanced marginally.

Since current algorithm works segment by segment, any violation for minimum radius of curvature (dog length severity) for a segment can be compensated by modifying coefficients. Even after modification the problem does not get rectified then it will be reported to the user. Since the coefficients are not so sensitive to radius of curvature so there may be a situation to change the input parameters (points, tangents, pre hold length and post hold length). Current observation also matches with Vassilev[9] and Park et al [10]. In these work [9,10] the third energy coefficient has not been considered. They have used global interpolation technique, which may not be relevant for well path application. In this work there are two straight elements in each segment with zero energy coefficient values, which help in maintaining linearity. Fang and Gossard[12] tried his algorithm for Z-shaped data points to get optimal number of elements. They have found that if the number of element increases it does not give smoother curvature. Less number of elements may give better smoothness. In this work, Hermite interpolation function has been used. Results shown are for 2D data points. In my opinion the iterative technique suggested by Fang and Gossard[12] is better to limit number of elements. I have obtained satisfactory results with three elements for each segment of the well. Curvature plots shows this fact (see Figures 4, 6 and 8). All the results have been implemented for 3D data points.

In the present work, three energy terms have been used which is giving smoother curvature than Vassilev[9] and Park et al[10]. In references $[9,10]$ only two energy terms have been used. In many applications it is needed to have a smoother curvature. Drilling industry can directly use results of this 
work. Output of this formulation gives a list of survey points. Observation of the results shows the importance of energy and fitting coefficients in smoothness of curve. When all four coefficients are present then effect of individuals is not so sensitive. All the results for well are drawn from the survey data points, which are final output for a drilling engineer.

\section{CONCLUSION}

Local minimum-energy, finite-element based formulation for well-path design has been performed in this work. The effect of energy and fitting coefficients on minimum energy well has been studied in this work. This work helps in iterative well design. The segment which is not satisfying drilling criteria can be interactively redesigned. Even making the energy coefficients zero for straight elements there are fitting errors coming due to the energy and fitting coefficients given for the curved elements. Magnitude of error is small and that can be controlled by modifying the coefficients. One reason for smaller magnitude of the error is that the input points are in two straight lines for each segment.

The cost analysis of the well for different length can be considered as the extension of the work. This work can be used in energy based surface fitting. Getting optimal coefficients are also an open issue for further research.

\section{References}

(1) Joshi, S D, 1991,“'Horizontal Well Technology “, 1991, PennWell publication

(2) Helmy, M W, Khalaf, F and Darwish, T A, 1997.

“Well Design using a Computer Model “, paper (SPE 37708), 42-47.

(3) Piegl, L and Tiller, W, 1995, "The NURBS book" New York, Springer

(4) Kjellander, J A P, 1983, "Smoothing of cubic parametric splines", Computer Aided Design, 15(3), pp-175-179.

(5) Kallay, M, 1987, "Method to approximate the space curve of least energy and prescribed length", Computer Aided Design, 19(2), 73-76.

(6) Lee, E T Y, 1990, "Energy, fairness and a counter example", Computer Aided Design, 22(1), pp37-40.

(7) Cheng, F and Barsky, B A, 1991, "Interproximation: interpolation and approximation using cubic spline curves", Computer Aided Design, 23(10), 700-706.

(8) Wang, X, Cheng, F, Barsky, B A, 1997, “Energy and B-spline interproximation”, 29(7), pp485-496.

(9) Vassilev, T I, 1996, "Fair interpolation and approximation of B-Splines by energy minimization and point insertion", Computer Aided Design, 28(9), 753-760. 
(10) Park, H, Kim, K and Lee, Sang-Chang, 2000, "A method for approximate NURBS curve compatibility based on multiple curve refitting", Computer Aided Design 32(20) 237-252

(11) Celniker, G, and Gossard, D C, 1991, "Deformable Curve and surface finite elements for free-form shape design", Comput Graph 1991, 26(2), PP 157-66.

(12) Fang, L and Gossard, D C, 1995, "multidimensional curve fitting to unorganized data points by non-linear minimization.", Computer Aided Design, 27(1), 45-58.

\begin{tabular}{|c|c|c|c|c|c|c|c|}
\hline \multirow{21}{*}{$\begin{array}{l}\text { Test } \\
\text { Example } \\
\text { Shown } \\
\text { In } \\
\text { Figure-7 }\end{array}$} & $\gamma 1$ & $\alpha$ & $\beta$ & $\gamma$ & $\begin{array}{l}\text { Total } \\
\text { Length }\end{array}$ & $\begin{array}{l}\text { Max } \\
\text { curvature }\end{array}$ & $\begin{array}{l}\text { Max } \\
\text { Error }\end{array}$ \\
\hline & \multirow{20}{*}{$1.0 \mathrm{e}^{6}$} & \multirow{10}{*}{0.0} & \multirow{10}{*}{0.0} & 0.0455 & 817.54 & .247607 & .0442646 \\
\hline & & & & 0.0855 & 817.357 & .247289 & .0469721 \\
\hline & & & & 0.155 & 817.039 & .246728 & .0517032 \\
\hline & & & & 0.455 & 815.678 & .244214 & .0722978 \\
\hline & & & & 0.855 & 813.899 & .240641 & .0997441 \\
\hline & & & & 1.055 & 813.024 & .238768 & .113371 \\
\hline & & & & 1.455 & 811.302 & .234873 & .140366 \\
\hline & & & & 2.055 & 808.789 & .228713 & .184756 \\
\hline & & & & 2.455 & 807.158 & .224438 & .221493 \\
\hline & & & & 2.955 & 805.167 & .218955 & .266617 \\
\hline & & \multirow{10}{*}{1.0} & \multirow{10}{*}{0.2} & 0.0455 & 817.283 & .247064 & .0481637 \\
\hline & & & & 0.0855 & 817.1 & .246742 & .0508914 \\
\hline & & & & 0.155 & 816.782 & .246174 & .0556486 \\
\hline & & & & 0.455 & 815.426 & .243633 & .0762861 \\
\hline & & & & 0.855 & 813.651 & .240027 & .103722 \\
\hline & & & & 1.055 & 812.778 & .23814 & .117333 \\
\hline & & & & 1.455 & 811.061 & .234219 & .144288 \\
\hline & & & & 2.055 & 808.555 & .228029 & .190092 \\
\hline & & & & 2.455 & 806.928 & .223739 & .226758 \\
\hline & & & & 2.955 & 804.943 & .218242 & .271794 \\
\hline
\end{tabular}

Table-1 (effect of $\gamma$ on well path) 


\begin{tabular}{|c|l|l|l|l|l|l|l|}
\hline $\begin{array}{l}\text { Test } \\
\text { Example }\end{array}$ & $\alpha$ & $\beta$ & $\gamma$ & $\gamma 1$ & $\begin{array}{l}\text { Total } \\
\text { Length }\end{array}$ & $\begin{array}{l}\text { Max } \\
\text { curvature }\end{array}$ & $\begin{array}{l}\text { Max } \\
\text { Error }\end{array}$ \\
\hline 1 & 0.0 & 0.0 & 0.0 & $1.0 \mathrm{e}^{6}$ & 3327.3 & 0.640471 & .0410935 \\
\hline 1 & 0.0 & 0.0 & 0.0455 & $1.0 \mathrm{e}^{6}$ & 2048.01 & 0.074923 & 0.083902 \\
\hline 1 & 1.0 & 0.2 & 0.0 & $1.0 \mathrm{e}^{6}$ & 1857.69 & 0.225023 & .108946 \\
\hline 1 & 0.2 & 1.0 & 0.0 & $1.0 \mathrm{e}^{6}$ & 1911.97 & .252363 & .0987742 \\
\hline 2 & 1.0 & 0.2 & 0.0 & $1.0 \mathrm{e}^{4}$ & 794.767 & .203723 & .51956 \\
\hline 2 & 1.0 & .20 & 0.0 & $1.0 \mathrm{e}^{6}$ & 817.493 & .247427 & .0450737 \\
\hline 3 & 0.0 & 0.0 & 0.0 & $1.0 \mathrm{e}^{6}$ & 2002.27 & .0851196 & $1.78 \mathrm{e}^{12}$ \\
\hline 3 & 0.0 & 0.02 & 0.0 & $1.0 \mathrm{e}^{6}$ & 1996.95 & .06137 & .0001570 \\
\hline
\end{tabular}

Table-2 (effect of $\alpha, \beta, \gamma$ and $\gamma 1$ on well path) 